\title{
MESA 4: LA VINCULACIÓN CON LO PÚBLICO Y LA UNIVERSIDAD DE CHILE
}

Alejandra Araya, Historiadora y Directora del Archivo Central Andrés Bello como moderadora.

Servet Martinez, Premio Nacional de Ciencias Exactas.

Maria Cecilia Hidalgo, Premio Nacional de Ciencias Naturales.

Inés Pepper, Magister en Biología y Profesora asociada de la Universidad de Chile.

María Eugenia Góngora, Decana de la Facultad de Filosofía y Humanidades (representada por Jennifer Abate, Periodista de la Vicerrectoría de Extensión y Comunicaciones). 


\section{MESA 4: "LA VINCULACIÓN CON LO PÚBLICO Y LA UNIVERSIDAD DE CHILE”}

Alejandra Araya: Buenas tardes a todos y todas. Sean bienvenidos a la última mesa del diálogo "Reforma y educación pública: Reflexión y debate desde la Universidad de Chile". La presente mesa nos convoca en torno a la vinculación con lo público y la Universidad de Chile, lo que parte de un planteamiento muy importante que tiene que ver con la misión de nuestra Universidad. De alguna manera, se nos convoca en torno a una tesis, que con ello tienen que ver las dos preguntas que se formularán a continuación a nuestros invitados.

Esa tesis sería, de alguna manera, que la Universidad de Chile tiene un deber con lo público, una misión, y ello se podría leer, por un lado, desde su aporte a la formación de profesionales y, por otro, en su labor de hacerse cargo de un problema: la desigualdad en Chile, tema que ha cruzado toda la discusión de hoy día en este diálogo. A nuestros invitados y panelistas les vamos a formular dos preguntas y vamos a iniciar con la primera que tiene dos partes: ¿Qué papel le corresponde a la Universidad de Chile en la formación de los futuros profesionales del país? y luego, ¿Cómo compatibilizar la excelencia académica con la igualdad de oportunidades? Vamos a iniciar con las palabras que envió por escrito la Decana de la Facultad de Filosofía y Humanidades, María Eugenia Góngora, y que van a ser leídas por Jennifer Abate.

Jennifer Abate: Buenas tardes a todos. Como decía Alejandra voy a compartir con ustedes las reflexiones que nos envió la Decana de Filosofía y Humanidades, María Eugenia Góngora:

La mesa en que tengo el honor de participar tiene como tarea responder dos preguntas que si bien tienen un carácter bastante general en apariencia, parecen ser más complejas de lo esperado en sus posibles respuestas. Responderé desde la perspectiva de las humanidades y la educación, los temas más cercanos a la Facultad que pertenezco y a la que he pertenecido desde que ingresé como estudiante al antiguo Pedagógico de la Universidad de Chile.

Desde su fundación, la Universidad de Chile forma profesionales de acuerdo a las necesidades del país y esa formación estaba, desde el comienzo, asociada a la reflexión, estudio e investigación. En ese sentido, creo que nuestra principal 
vinculación con el medio es esa formación que por cierto ha ido evolucionando y acogiendo las nuevas necesidades del país.

Desde sus orígenes, la Universidad de Chile ha mantenido una estrecha vinculación con la construcción de la República, lo que ha hecho de esta formación de profesionales una actividad con un sello característico. A lo largo de esta historia, la Universidad ha acompañado su tarea de formación de profesionales con su sentido de vinculación a los problemas de nuestra sociedad y creo que gracias a este sentido de pertenencia se ha trasmitido hacia los profesionales de la Universidad de Chile un sentido de compromiso que ha constituido una impronta que ha marcado esta tarea formadora y le ha dado a esta un sentido más trascendente.

Al mismo tiempo, la Universidad ha desarrollado una actividad científica muy importante, la que tiene actualmente un componente que, dada la existencia a fondos públicos ajenos a las universidades, exige que no sólo los proyectos propiamente de investigación, sino también otras iniciativas académicas, se deban financiar mediante la postulación de fondos externos. Es una realidad a la que ya estamos completamente acostumbrados, pero que evidentemente ha burocratizado nuestra actividad en este campo de manera importante y también ha resultado en la necesidad de dedicarse a la gestión de los proyectos, más allá de la propia investigación, lo que hace más difícil que los profesores, o algunos de ellos al menos, puedan dedicar un mayor tiempo no sólo a la docencia, sino también a la tutoría y mentoría de los estudiantes, una dedicación que estos reclaman con fuerza.

Me parece que esta es una práctica que debiéramos reconstruir y crear los espacios y el tiempo para este diálogo libre y formativo entre el profesor y el estudiante. Hay que reconocer también que en nuestro medio es actualmente la investigación la que otorga un mayor prestigio académico y que, dadas las condiciones de la carrera académica, son los proyectos ganados y las publicaciones los que tienen un peso relevante tanto para los académicos como para la institución.

La segunda parte de esta pregunta, la compatibilidad de excelencia académica e igualdad de oportunidades, es más compleja que la primera. Me parece que aquí existe un desafío especialmente importante para la Universidad en los próximos años. Chile ha seguido una trayectoria singular en materia de masificación de la educación superior, pues esta se ha hecho básicamente por la vía de la expansión de la oferta privada y se espera que en los próximos años y con una revitalización de la educación pública, este proceso se revierta en parte y presenciemos una expansión de la matrícula en las instituciones de educación superior públicas, incluida la Universidad de Chile. Ello implicaría mayores desafíos para la docencia universitaria, pues será necesario trabajar con jóvenes con un capital cultural restringido. Una apertura a la equidad y una mayor masificación de las universidades 
públicas, planean importantes desafíos para mantener los niveles de excelencia que la Universidad de Chile se plantea.

Con respecto al tema de la igualdad de oportunidades, hay que reconocer que todo el sistema escolar, con la posible excepción de la educación básica y también, por cierto, con la educación superior, está construido sobre la desigualdad y sobre un sistema de evaluación permanente. Por lo demás, en la educación superior y en lo que se refiere a los académicos, la jerarquización académica supone que nuestra Universidad es esencialmente meritocrática. La evaluación de los méritos de cada cual corresponde a los criterios de evaluación que han ido cambiando también con el tiempo. Y si bien es cierto que se puede observar una preocupación creciente por la validez de los criterios de evaluación en ciertas actividades que no corresponden a las ciencias llamadas duras, por ejemplo, en las artes, en los hechos y como en todo sistema competitivo, en la carrera académica hay ganadores y perdedores, diciéndolo de una forma cruda, pero realista. A partir de esta constatación me parece imprescindible que el rigor académico se construya y evalúe reconocimiento según la especificidad y la naturaleza diversa de los saberes y disciplinas que se cultivan en la universidad.

Alejandra Araya: Muchas gracias, Jennifer. Vamos a continuar con la Profesora Inés Pepper para hacer un contrapunto entre el área de las humanidades y el ámbito de la salud y la biología, que es de donde proviene la Profesora Pepper.

Inés Pepper: Buenas tardes. Quisiera partir esta conversación con algunos recuerdos personales: yo estudié en Chile en el Colegio Alemán y cuando se terminaba el año iban desde el Liceo 7 a tomarnos el examen final y si no pasábamos este examen, lamentablemente teníamos que hacerlo de nuevo en marzo. El otro recuerdo es el siguiente: cuando trabajé en la Facultad de Medicina no pagué nunca ningún peso, ningún tipo de matrícula ni aranceles, en el contexto de un país mucho más pobre que el de ahora. Es decir, si hablamos del desarrollo que ha tenido el país y nos llenamos la boca con que somos un país desarrollado, de la OCDE, uno podría decir, ¿cómo fue posible que estudiáramos gratuitamente en la Universidad de Chile? y ¿cómo un colegio de una colonia bastante poderosa, desde el punto de vista monetario, tuvo que cumplir con una norma que a mí me parece correcta, dar un examen frente a la institución estatal del país donde uno está educando?

Volviendo a la pregunta respecto a qué tipo de profesionales debe formar la Universidad de Chile, me quiero referir a algunos temas en específico, pues el tema en sí es bastante amplio. Yo creo que un problema muy importante tiene que ver con que nuestros profesionales tengan la vocación, interés y capacidad de participar en el desarrollo integral del país y eso es importante, porque en este momento -así 
lo percibo en la Facultad donde hago docencia- la vocación no siempre es la que aparenta ser, ya que muchos de los estudiantes quedan en alguna carrera debido a que el sistema de selección está mal concebido y su interés no es el suficiente para que después ejerzan con la vocación que se requiere.

Además, y dadas las características sociales del modelo en el que estamos viviendo, existe en los estudiantes un compromiso más fuerte consigo mismo que con la sociedad y ese tema es bastante difícil de superar, por lo menos en la Facultad de Medicina. En algunas carreras eso es mucho más notorio que en otras, pero en nuestro caso hay muchos estudiantes que estudian Medicina porque su interés es trabajar en una clínica privada, tener dinero, prestigio y ser líder. No todos son así, pero hay bastantes que presentan esa característica.

Ahora bien, creo que la pregunta clave es si nosotros queremos formar profesionales técnicamente aptos para resolver problemas de personas, de sociedades, problemas de salud, educacionales o si debemos ir más allá. Yo creo que una de las grandes falencias que hay en todos los programas de estudio que me ha tocado ver es la falta de compromiso con lo más amplio: enseñamos cosas que son técnicamente muy apropiadas, pero falta la visión del ser ciudadano y eso no sé cómo se puede enseñar de manera precisa. Personalmente creo que, por ejemplo, el hecho de que hayan sacado la Educación Cívica de los colegios es un tema más o menos potente que hace que nuestros estudiantes muchas veces no tengan ninguna idea al respecto. Y creo que la Universidad de Chile tiene como uno de sus deberes formar ciudadanos comprometidos con el medio ambiente que los rodea.

El otro tema que me gustaría plantear se refiere a la formación de nuestros académicos(as). Básicamente, quiero referirme a la necesidad de homogeneizar la carrera académica en las diversas Facultades y el país, pues creo que debe haber una carrera académica nacional a la que pertenezcan todas las personas que participan en la investigación, en la docencia y extensión, y que un profesor asociado de una universidad debe ser el equivalente a un profesor asociado en otra universidad, tanto en la capital como en regiones. Una carrera académica nacional es importante, porque así se puede producir mucho mejor la movilidad entre una universidad y otra y va a haber un nivel de enseñanza más o menos similar en distintas universidades estatales, por lo menos en el ámbito de lo estatal debería ser así.

Otro tema que es muy relevante en relación al tipo de profesional que tiene que formar la Universidad de Chile tiene que ver con el financiamiento de la Universidad. Creo que todas las universidades estatales deben ser financiadas completamente por el Estado, vale decir, que los académicos y los funcionarios de estas universidades deben ser funcionarios públicos pagados por el Estado y no por el estudiante. A mí me da vergüenza recibir todos los meses un sueldo que ha sido pagado por los estudiantes a los que les estoy enseñando, porque pasan a ser mis sostenedores o 
clientes. Creo que la Universidad de Chile debe hacer todos los esfuerzos posibles para convencer al gobierno de que, en tanto Universidad estatal, tiene que ser financiada $100 \%$ por el Estado.

Alejandra Araya: Muchas gracias Inés. Ahora le damos la palabra a Cecilia Hidalgo quien, desde las ciencias naturales, nos entregará nuevas aristas sobre este tema.

María Cecilia Hidalgo: Yo comparto bastantes puntos con lo que acaba de plantear Inés. También les quiero contar algo de mi formación, que siempre ha sido en el ámbito público: yo estudié en un liceo muy malo que ya no existe, sin embargo, no fue lo suficientemente malo para cortarme las alas, porque después pude ingresar a la universidad. Pero sobreviví a esa experiencia y estudié primero bioquímica y después, en una decisión bastante poco convencional, opté por hacer el primer doctorado en Ciencias en la Facultad de Ciencias de la Universidad de Chile. Respecto a la pregunta, ¿cómo puede contribuir la Universidad de Chile o cuál debe ser el papel de la Universidad en la formación de profesionales?, a mí me gustaría ampliarla un poco, porque la Universidad no sólo forma profesionales, sino que forma gente que trabaja en muchos ámbitos creativos (artistas, gente que trabaja en ciencias sociales, filósofos, científicos que están en las áreas de ciencias naturales o los que trabajan en matemáticas o en física teórica). De alguna manera, todo gran desarrollo que ha tenido la ciencia en Chile, y en eso la Universidad de Chile ha tenido un papel central de liderazgo, ha pasado como desapercibido. Porque todavía no logramos convencer a la gente que toma las decisiones o a aquellos que tienen el poder en nuestro país, de que la ciencia que se hace en Chile es de muy buen nivel y reconocida internacionalmente.

Además, hay otro aspecto que a mí me gustaría destacar y que guarda relación con el hecho de que, si bien el ambiente en ciencias es bastante competitivo, el quehacer no discrimina como se discrimina en otras áreas de nuestro país, porque no hay discriminación social. Eso es algo bastante sorprendente, porque yo constato que mucha de la gente que está en mi grupo viene de situaciones familiares bastante precarias y, sin embargo, son científicos brillantes. Yo creo que es criminal que un país no le dé a toda su gente joven la oportunidad de desarrollar al máximo sus talentos. Eso no significa que todas las personas vayan a ser iguales, porque algunas personas tendrán más talento innato o tendrán más capacidad de esfuerzo, de trabajo y podrán llegar más lejos en su quehacer. Lo que no es admisible, a mi parecer, es que se los discrimine de partida. Yo tengo una visión de que lo que estamos tratando de hacer aquí, es derribar o perder esta sociedad tan centrada en lo material que tenemos. 
Yo me acuerdo de haber crecido en un país distinto, en el cual se valoraba la cultura, se valoraba la gente que tenía distintas profesiones o que era profesor universitario, por ejemplo. Ahora lo que más se valora es quien tiene más estatus material, porque hemos copiado la parte negativa de la sociedad norteamericana, en la cual yo viví muchos años, pero no hemos copiado la parte más positiva de valoración de cultura. Porque la sociedad norteamericana tiene estos dos aspectos: un materialismo extremo, pero, al mismo tiempo, una filantropía y un apoyo a las ciencias y a las artes, cuestión que en este país no se da. Entonces, yo creo que estamos en el camino de tratar de cambiar esta visión tan mercantilista de la sociedad. En ese sentido, comparto con Inés el optimismo en el futuro y creo que la gente joven nos ha dado una gran lección en su lucha por tratar de establecer la idea de que la educación es un derecho para todos y que es inadmisible hacer este tipo de discriminación.

Alejandra Araya: Muchas gracias, Cecilia. Para completar esta primera ronda, le doy la palabra a Servet Martínez, Premio Nacional de Ciencias Exactas.

Servet Martínez: En primer lugar, sobre la excelencia y la calidad, quiero remitirme a una anécdota que me ocurrió hace casi 40 años, en el año 1976. Yo estaba en París en mi primer año de llegada a estudiar matemáticas y me invitaron a una peña, donde había mucho chileno exiliado. Ahí estaba el Gitano Rodríguez, autor de Valparaíso, que empieza a hablar de una conversación que tuvo con Paco Ibáñez, cantante catalán que se exilió en Francia y que vino a Chile en la época 19701973. Entonces el Gitano Rodríguez cuenta que le preguntó a Paco Ibáñez por qué cantaba solamente canciones de Luis Felipe, del Arcipreste de Ita y no sus propias canciones. A esto Paco Ibáñez le responde: "Nosotros, cantantes bardos, somos los que alimentamos al pueblo, y si al pueblo le damos basura, el pueblo basura come". Vale decir, si uno no entrega calidad, si uno entrega algo que no es de primera calidad, ese algo que no es de primera calidad se consume, se enseña, se trabaja y ese es el techo de nuestros estudiantes. Lo anterior es un punto que a mí me gustaría que quede claro y que se ha dicho anteriormente sobre la reforma: "Si calidad es sin calidad, entonces no tiene sentido la reforma". Lo que entregamos es consumido, analizado y usado. Son las herramientas que van a usar los profesionales, científicos, humanistas y los estudiantes en nuestro país, de modo que creo que ese es un punto absolutamente crucial.

Dicho esto, y en relación a la calidad y la equidad, cabe aclarar que ambas son construcciones sociales que no existen fuera del tiempo y de la sociedad. Ambas existen en un tiempo y en sociedades en un minuto dado, tal como existían en la época de 1970-1973 o en los años sesenta, cuando un porcentaje muy ínfimo de la 
población estudiaba en la universidad, porcentaje relativamente pequeño integrado por los más meritorios. Hoy en día estamos frente a otra sociedad más compleja; una sociedad con una cantidad inimaginable de recursos invertidos en ingeniería y en progresos técnicos.

Con Jaime San Martín, aquí presente, hemos hecho un estudio de cómo funciona la concentración. Por ejemplo, en ciencias hay concentraciones en torno a un tópico específico, como biomedicina. Por otra parte, toda la ciencia que se hace en Chile corresponde a un cuarto de lo que invierte el Estado de Iowa, de tres millones de habitantes. Entonces, la pregunta es cómo conjugamos la concentración extraordinaria de recursos con la equidad. Esta es una tarea que, para abordarla de manera real, tenemos que abordarla de frente, con los datos y números claros, porque hay que mirarlos, analizarlos y tratar de comprenderlos. Si Estados Unidos tiene una producción científica del 40 o 30\% de la producción, en capacidad de cómputos tiene más del 50\%. Luego, si nos vamos a grandes computadores tienen muchos más y Brasil, en vez de ser el 3\% de la producción mundial, en capacidad de cálculo tiene el 0,4\%. Vale decir, hoy tenemos que decir cómo nos planteamos frente a esto como país, cómo nos planteamos y cómo conjugamos calidad con equidad, mirando el hecho de que calidad y equidad son también construcciones sociales que tenemos que decidir cómo son y, en relación a esto, dónde invertimos, cómo lo hacemos y cómo incorporamos.

Sobre lo público y lo privado me interesaría contar otra anécdota. Una vez en Japón estaba conversando con un Decano de Ciencias Sociales para Ciencia Avanzada y Tecnología y me dijo la siguiente frase: "Los japoneses somos todos iguales, no tenemos una capacidad inventiva, somos todos muy obedientes, por eso necesitamos un gobierno de derecha. Y los chinos son unos individualistas terribles y por eso necesitan un gobierno de izquierda". Con esto, lo que trataba de decirme el japonés es que el juego entre lo público y lo privado guarda relación con aquellas construcciones que deben ser incentivadas, reflexionadas y construidas. Para él la construcción de lo público era un problema de la educación del pueblo durante años y del gobierno, incentivos también para construir lo público así como para construir lo privado.

En relación a esta anécdota, lo público y lo privado son construcciones que tenemos que hacer en Chile. Obviamente, aquí hay un desbalanceo hacia lo privado, siendo lo público aquello que necesita ser reflexionado, pensado y actuado. Creo que, hoy en día, la Universidad de Chile tiene la oportunidad de avanzar en esta construcción de un verdadero referente público, fuerte, basado en la ciencia y en todas las ciencias, es decir, en las ciencias humanas, humanidades, matemáticas y ciencias naturales, pero para eso debemos mirarla realmente con la verdad. 
Alejandra Araya: Muchas gracias, Servet. Ahora pasamos a un segundo tema, que tiene que ver con cómo la Universidad de Chile va a aportar a un país más igualitario. Me voy a permitir hilar algunas cuestiones que me parecen relevantes en relación a lo que nos han planteado: por una parte, estamos hablando de modelos de desarrollo del país y me parece muy significativo lo que han señalado respecto a que existe un relato y que la misión de la Universidad de Chile y su vinculación con el medio es una forma de comprender la relación con otro, que es esta entidad mucho más colectiva y nacional y es ahí donde están los problemas, porque todas las metas han sido enfáticas en declarar que hay un país que ya no existe. Ustedes mismos hablan como si vinieran de un país totalmente ajeno a lo que hay y, por lo tanto, desde esa realidad la Universidad de Chile vuelve a retomar esta misión de recuperar una cierta mística de la formación de los profesionales, que sobrepase lo meramente técnico y la calidad, porque la Universidad de Chile no puede pensarse como un sujeto anómalo. En ese marco, debemos comprometernos con el medio como una responsabilidad social con ese otro que es nuestro país, nuestra sociedad, esto es, una nueva misión frente a la realidad de un país profundamente desigual. Dicho esto, continuaremos leyendo lo que comenta la Decana María Eugenia Góngora a partir de la pregunta: ¿Cómo puede aportar la Universidad de Chile a un país más igualitario?

Jennifer Abate (en representación de María Eugenia Góngora): Con respecto a la segunda pregunta, la respuesta también es difícil de contestar, porque creo que si bien muchos de nosotros tenemos en el horizonte el significado de la palabra igualdad o cómo podemos construir un país igualitario, sospecho que los caminos para alcanzarla son ásperos y difíciles, tal como se puede observar ahora mismo en relación a la discusión de la Reforma Educacional. Por lo mismo, resulta instructivo ver que en nuestro país el tema de la igualdad ha sido un deseo y una aspiración difícil de alcanzar.

En su Filosofía de la educación, publicada en el año 1927, don Valentín Letelier menciona prácticamente los mismos temas de discusión que ahora nos preocupan. Como buen pensador positivista y anticlerical, su preocupación es la influencia excesiva de la iglesia, pero encontramos también allí temas como la enseñanza sujeta a la oferta y la demanda y la gratuidad de la enseñanza, gratuidad para todos, dado que se trata de un bien público, la libertad de la enseñanza y otros semejantes. Al mismo tiempo, hay que reconocer que la educación, aun con sus limitaciones, es un tema fundamental en asociación con la igualdad de los ciudadanos. Creo que en las Facultades de Filosofía y Humanidades, Ciencias y Ciencias Sociales, existe una reflexión, una práctica, en formación de profesores desde hace ya muchos años, además de una serie de experiencias valiosas que apuntan en ese sentido particular. 
Un ejemplo muy concreto es el trabajo que se ha realizado desde la Facultad de Ciencias con jóvenes que están internos en hogares del Sename y que en forma de taller han continuado como experiencia de formación para nuestros estudiantes de pedagogía. En suma, pienso que una perspectiva esencialmente emancipadora y no tecnocrática de la formación docente y de la educación son relevantes para la construcción de un país más igualitario. Por otra parte, sabemos que en todas las unidades académicas de la universidad existe una preocupación por este tema y múltiples iniciativas que son una muestra de la importancia de este asunto en nuestra universidad. Veo, además, que en un futuro cercano se empieza a producir una consolidación de lo ya construido y un amplio desarrollo del tema de la educación y de las pedagogías en la Universidad de Chile, lo cual será un gran aporte de la Universidad al proceso de la reconstrucción de la educación pública en nuestro país.

Alejandra Araya: Muchas gracias, Jennifer. A continuación, la Profesora Inés Pepper nos entregará su perspectiva sobre esta problemática.

Inés Pepper: En primer lugar, creo que la Universidad de Chile puede aportar muchísimo más de lo que pensamos normalmente a la construcción de un país más igualitario. Esto puede ser visto desde dos ámbitos que la Universidad actualmente desarrolla: el ámbito de la formación de pre y postgrado y el ámbito de la investigación en el área de las ciencias, humanidades, arte, etc. En el primer caso, yo creo que podemos aportar a un país más igualitario si damos la oportunidad que no tienen todos los estudiantes que desean continuar con sus estudios en la Universidad. Ese es un tema bastante difícil y me imagino que nuestra Vicerrectora Académica debe estar muy consciente de eso, puesto que necesariamente tiene que haber algún sistema que permita homogeneizar a los estudiantes, independientemente de qué educación previa tienen. Eso lo hemos vivido con bastante intensidad últimamente en nuestra Facultad, donde han entrado estudiantes a partir de otros sistemas que no son PSU y realmente es una cosa difícil, pero no imposible de lograr. Su rendimiento ha sido bueno en la medida que hemos sabido acercarnos a ellos y tener un contacto más directo.

En relación a esto, quiero entrar en otro punto que pienso es muy importante para la Universidad de Chile: nuestra relación como académicos y docentes con nuestros estudiantes; ¿cuánto conocemos de ellos?, ¿cuánto nos acercamos a ellos? Y yendo más allá de lo académico, ¿cuántas veces conversamos con ellos en el pasillo o nos tomamos un café?, ¿qué problemas tienen?, ¿cómo se comunican? Si uno compara un estudiante de hace 10 o 15 años, o quizás más, con un estudiante actual, son personajes totalmente diferentes en términos de las herramientas que utilizan para comunicarse y de los métodos que utilizan a través de toda la tecnología 
nueva que hay. Es impresionante cuando uno está, por ejemplo, haciendo una clase y en el descanso los estudiantes, en vez de conversar o salir a comerse un sándwich, están con su teléfono y no hablan. Entonces es una realidad distinta a la de hace diez años y, que yo sepa, la Universidad de Chile no la ha estudiado en profundidad. Hay una manera de comunicarse de nosotros, los académicos, hacia ellos. Nosotros hacemos una clase, subimos el PowerPoint al aula digital y si tú le preguntas algo que no está en el PowerPoint, para ellos no está la información. De modo que hay un tema con información, formación y comunicación que yo creo que hay que investigar urgentemente.

Ahora, en cuanto al académico y su labor de investigación, creo que la Universidad de Chile puede colaborar con la construcción de un país más igualitario si es que toma o le da especial atención a los problemas país, a los problemas que tienen que ver con el desarrollo integral del país, llámese nuevos sistemas de salud que sean diferentes a la Isapre y Fonasa, nuevos sistemas de previsión diferentes a la AFP y el INP y nuevos sistemas de comunicación. Es inconcebible que la Universidad de Chile no tenga su propio canal de TV. Cuando uno ve las encuestas que se hacen en algunos programas donde va la gente común y corriente, y le preguntan: “ $¿ T i e n e$ frío?”, “¿qué se pone usted para combatir el frío?”, y luego están 20 minutos hablando de cómo combatir el frío, entonces uno dice: ¿En qué país estoy viviendo? En ese sentido, yo creo que hay que entregar cultura y hay que hacerlo ya.

Alejandra Araya: Muchas gracias, Inés. Ahora, Cecilia Hidalgo nos dará su opinión sobre este tema.

Cecilia Hidalgo: Creo que Alejandra tiene razón cuando plantea que este país ha cambiado mucho. Realmente es impresionante cómo se ha estratificado nuestra sociedad y cómo, de alguna manera, se mira muy en menos a la persona que es pobre y que viene de una etnia distinta. Aquí hay un grupo de gente que cree que sabe todo y que maneja el país y yo creo que la Universidad de Chile puede, efectivamente, contribuir a hacer un país un poco más igualitario, algo que va a tomar mucho tiempo si sigue llevando el estandarte de que la cultura (y yo la ciencia la pongo como parte de la cultura de un país), es algo importante, es un valor que le da dignidad a las personas. Ese es un papel muy importante de la Universidad de Chile y digo con mucho orgullo que, pese a todo lo que se la ha atacado, nuestra Universidad es todavía líder en muchos ámbitos de la ciencia y la cultura en el país.

Por ejemplo, la Universidad de Chile tiene muy pocos fondos. En el caso de las becas, no tiene para sus estudiantes y tiene que competir por las becas de postgrado en competencias nacionales. Además, tiene que competir por todos los proyectos importantes en competencia nacional y seguimos siendo, pese a todo, la 
Universidad número uno en estos concursos. Entonces algo tienen los académicos de la Universidad de Chile que han logrado sobrevivir y no sólo sobrevivir, sino que hacerlo muy bien en este sistema, que claramente ha tratado de jibarizarla. Creo que quizás sería importante poner un poco la mirada hacia dentro y tratar, desde la Universidad, de recuperar lo que esta fue, abordando los problemas que son verdaderamente importantes para el país. Yo veo que, en mi concepción, la ciencia es un continuo; tenemos desde la ciencia más básica hasta la más aplicada, además de tecnología e innovación. La gente que va a hacer los desarrollos más aplicados tiene que tener una buena formación básica para poder ser competente. A mí me importa muchísimo dónde se forma la gente que va a hacer las carreras más técnicas en el país. Los centros de formación técnica realmente no están formando a la gente con todas las capacidades que debería. Por ejemplo, un país como Alemania, que valora mucho un técnico capacitado, aquí se mira como un ciudadano de segunda clase; no es un profesional, sino un técnico, y mientras no cambiemos esa cultura, no vamos a avanzar mucho como país, porque seguimos segregando a la gente por el tipo de actividad que desarrolla. Si somos todos importantes en la sociedad, somos importantes los filósofos, los artistas, los científicos, los médicos, los ingenieros y las personas que están en ámbitos muchos más técnicos.

Todos nosotros tenemos que contribuir a que este país sea un lugar mejor y abordar problemas, por ejemplo, relativos al campo de la energía, medio ambiente y envejecimiento de la población. Nosotros somos una paradoja, porque somos un país pobre que está envejeciendo como país rico y tenemos muchos problemas que se van a derivar de eso a muy corto plazo. Luego, ¿qué pasa con la obesidad?, ¿cómo es posible que nuestros jóvenes y niños estén alcanzando índices de obesidad que son muy alarmantes? Estos son problemas bien serios y que también nacen de la desigualdad y la segregación, porque es mucho más fácil comer comida chatarra, que es más barata y de más fácil acceso, que darle una alimentación más sana. A esto se suma que nuestros niños no hacen suficiente actividad física, aun cuando la necesitamos, sobre todo ahora que estamos sentados en el computador mirando el aparatito. Eso va en desmedro de la salud futura de toda nuestra gente joven.

Alejandra Araya: Muchas gracias. A continuación, el Profesor Servet.

Servet Martínez: Quiero partir con algo que decía Inés Pepper después del desastre de ver TVN o cualquier TV. Creo que una pregunta central es: ¿Por qué no somos más ignorantes de lo que somos? Se ha construido una ciencia y hemos construido muchas cosas; Chile se ha atrevido a hacer una experiencia social que es única en el mundo: un pueblo digno que construyó realmente su trayectoria en derechos humanos durante 17 años de una dictadura feroz. Por qué entonces, a pesar de 
todo, hoy en día y por una serie de aventuras como el movimiento estudiantil, ¿por qué no somos más ignorantes de lo que somos?

Yo creo que está probado en Chile que los excluidos, todos los niños excluidos, son equivalentemente inteligentes a los nuestros. Como decían Los Prisioneros "O somos tontos, somos ricos, pobres, qué sé yo". ¿Cuál es el punto sobre la educación? Aquí aterricemos sobre la igualdad y la educación; a mí me parece que la pregunta crucial es la igualdad de la educación dentro del contexto en el que esté. Yo creo que lo que quieren los excluidos es no ser excluidos, quieren participar y competir fuerte. En este punto, quiero contar otra anécdota de Armando Uribe en un seminario sobre el golpe de Estado en el año 1977. Él cuenta que escuchó que el Presidente Frei habría dicho que una constante histórica del Estado chileno es que los indios no crucen el Biobío y que el golpe se produjo porque los indios habían pasado el Biobío. Los pelos duros, el cabro de pelo duro, fue castigado brutalmente, pero acá, lo que ese cabro quiere, es oportunidad. Yo no entiendo por qué en la reforma educacional no se han volcado la mayor parte de los recursos, ahora mismo, en las escuelas municipales.

Como se dijo acá, cada vez que a la Universidad de Chile le ha tocado competir, le ha ido muy bien. En la enseñanza pública, cada vez que le toca competir a los estudiantes, les va a ir bien si es que los dejamos correr con las dos piernas, si es que no los dejamos cojos. Creo que esa es la esencia de la igualdad, pero como dijo algún antropólogo: "La igualdad no es un estado en que nada se mueve, la igualdad es un estado muy dinámico, de mucha competencia, muy áspero y riguroso" y esa es la verdadera igualdad. Allí donde los colegios municipales y las universidades estatales estén realmente compitiendo y con calidad. Ese era, en parte, el Chile de los años sesenta en una escala un poco más pequeña, pero esos eran los colegios fiscales. No era que los colegios fiscales dijeran: "no vamos a competir", sino que la gente, sus estudiantes y profesores, decían ser los mejores y por algo se decían los mejores; no era una palabra vana, era una palabra real.

Dicho esto sobre la igualdad, quisiera decir un par de puntos, porque yo no le creo a la Universidad de Chile cuando dice: "Estamos tratando de hacer lo mejor posible", no le puedo creer. El año 2002 la Ministra Mariana Aylwin había propuesto que el Pedagógico volviera a la Universidad de Chile cuando había una huelga gigantesca en el Pedagógico. Ella lo propuso y yo me acuerdo que en ese entonces llamé a la que era su subsecretaria, Pilar Armanet, y le dije: "Me parece una fantástica idea que el Pedagógico vuelva a la Universidad" y Pilar Armanet me dijo: "Servet, hay gente de tu propia Facultad que no quiere esto". Yo no sé qué fuerzas se habrán movido entre querer y no querer en ese minuto, pero eso nunca se ha analizado. Entonces yo tengo mis dudas con la Chile, porque en el día de hoy se planteó que la educación es un problema crucial del país y, aun así, la Chile no es 
capaz de crear la Facultad de Educación, siendo que desde el 2011 lo único que se quiere es que haya educación y hasta donde entiendo una manera muy interesante de que la haya es, obviamente, mediante una Facultad de Educación. Entonces, ¿por qué tengo que creer que las autoridades y académicos de la Universidad de Chile están tomando las decisiones pensando en Chile?

El último punto que quiero decir, entre calidad, equidad e igualdad, es el siguiente: ¿cómo hacemos toda esa construcción social? Tenemos que echarnos el país al hombro y eso es un desafío que está pendiente. A mí me pareció fantástico el movimiento estudiantil del 2011, pero nunca le he visto la voluntad de echarse el país al hombro. Yo nunca vi en el movimiento estudiantil alguien que dijera: "Nosotros, generación 2011 o 2012, nos vamos a echar el país al hombro". Si mal no recuerdo, la FECH y los movimientos estudiantiles en los años setenta lo que buscaban era justamente echarse el país al hombro. Yo nunca he visto al movimiento estudiantil, este año, salir en pro de algo. Hay autoridades que dicen: “Lo que queremos hacer es esto, esto y esto en la educación". Yo coincido con lo que los estudiantes decían que había que hacer, pero nunca he visto al movimiento estudiantil que diga: "Nosotros vamos a salir a apoyar eso". En los años sesenta y setenta, la gente era suficientemente generosa, no para apoyar lo de uno mismo, sino que también para apoyar lo de los otros.

Alejandra Araya: Bueno, muchas gracias Servet y muchas gracias a todo el panel. A modo de cierre, quiero quedarme con la imagen de la necesidad de una épica para un nuevo tiempo, porque también es fácil caer en los mitos autoconstruidos. En la inflexión histórica en la que estamos, desde la historia uno puede decir: "Las cosas han sido siempre igual, así que es difícil que cambien" o bien puede decir: "En realidad todo puede cambiar si hay voluntad de que cambie". Con eso estamos, además, parafraseando a un gran historiador inglés, Eric Hobsbawm, cuando decía: "Estamos en el fin del siglo XX largo y en el inicio del siglo XXI corto". Y, ¿ंen qué estamos?, ¿en qué inflexión está la Universidad de Chile de cara a la necesidad de una nueva épica? De cara a la realidad con una frase que dijo Cecilia: "Somos pobres, envejeciendo como ricos". Yo creo que deberíamos tomar esa metáfora desde ese mundo de la biología, de la materialidad de la existencia, como un elemento significativo de la reflexión sobre qué tipo de desarrollo hemos estado construyendo en el último tiempo y cómo nos vamos a echar el país al hombro. Quizás podríamos partir por echarnos primero la casa al hombro, que es esta Universidad a la cual igual interpelamos permanentemente. Agradezco a nuestra nueva Vicerrectora de Extensión y Comunicaciones la organización de este diálogo, la presencia de los asistentes y de nuestros invitados, con quienes esperamos mantener este diálogo abierto de cara al nuevo siglo XXI. Muchas gracias. 